Cerebral blood flow in diabetes mellitus

SIR,-Dr P Dandona and his colleagues (29 July, p 325) appear to conclude from thei most interesting demonstration of altered reactivity of the cerebral vasculature to $\mathrm{CO}_{2}$ in diabetics that these patients have an "inability to compensate with increased cerebral blood flow when metabolic requirements of the brain demand this." Such conclusion is almost certainly not justified.

Although $\mathrm{CO}_{2}$ has been suspected as a factor in the mechanism which couples blood flow to metabolism in the brain since Roy and Sherrington's classic study, ${ }^{1}$ no direct evidence exists for this supposition and recent evidence is against it. ${ }^{2}$ Failure to react to one specific stimulus can surely not be taken as evidence of an impaired ability of cerebral vessels to dilate under any circumstances. They may yet respond to the factors, as yet unidentified, which do couple blood flow to metabolism in the brain.

Physiological Laboratory, Cambridge

R M GARDINER

Roy, C S, and Sherrington, C S, fournal of Physiology, $1890,11,85$.

Cerebral Vascular Smooth Muscle and its Control. Ciba Foundation Symposium No 56. London,

\section{Misuse of hypnosis}

SIR,-With the increased interest in the use of hypnosis may I, through the courtesy of your columns, make some points concerning its use by unqualified persons for the purposes of entertainment or by lay therapists? Thes are as follows.

(1) No medical history is taken, and medical or psychiatric conditions can be present which could be aggravated by the hypnotic suggestion.

(2) Anxious patients could become more anxious or even depressed.

(3) Depressed patients could become more depressed and the possibility of suicide should not be overlooked.

(4) The psychotic person could become even more psychotic.

(5) The use of hypnosis is a psychotherapy and as such should only be used by those properly trained, and whenever practised it should be done in a setting where adequate psychiatric safeguards are provided.

(6) Conditions such as cerebral tumours, neurological disorders, hypoglycaemia, thyroid dysfunction, and drug dependence can only be recognised by the medically trained. All these illnesses may present psychological symptoms which the unwary and unqualified hypnotist may meet.

(7) The use of hypnosis can be easily abused. For example, subjects can be made to perform im moral or improper acts.

(8) The well-known hypnotic phenomenon of catalepsy is often utilised in performances of hypnotism, and orthopaedic problems may result.

(9) Doctors and dentists are bound by a code of thics which safeguards public and patients against abuses in the use of hypnotics. It is they alone who can assume full clinical and legal responsibility for their patients; unqualified persons are not so bound.

(10) The ability to recognise mental illness is essential so that the necessary treatment with drugs can be instigated. Non-qualified persons may not prescribe and have no knowledge of such treatment.

The report of a subcommittee of the BMA's Psychological Medicine Group Committee on "The Medical Use of Hypnotism"' states: "When it is used without proper consideration on persons predisposed ... to severe psychoneurotic reactions or antisocial behaviour... the commission of crimes involving even danger to life is not entirely to be ruled out." It emphasises that the relationship between hypnotist and subject may result in the release of powerful emotions which could only be recognised and controlled by properly qualified person. It therefore recommends that "the use of hypnotism in the treatment of physical and psychological disorders should be confined to persons subscribing to the recognised ethical code which governs the relation of doctor and patient."

The use of hypnotism by unqualified persons and for amusement debases and devalues a valuable therapeutic aid, while the use of hypnosis in cabaret turns and on the stage is degrading to human dignity.

D WAXMAN President,
Hypnosis,

London W Section of Medical and Dental Hypnosis,
Royal Society of Medicin

${ }^{1}$ British Medical fournal Supplement, 1955, 1, 190.

\section{Pigeon fanciers and disease}

SIR,-I naturally read with interest the paper by Dr D J Hendrick and others on budgerigarfancier's lung (8 July, p 81), and was pleased to feel substantially responsible for the fact that the "pigeon-racing community" is "medically well informed." As well as regular warnings in our magazines we have, of course, set up a research fund which looks into diseases of both pigeons and pigeon fanciers.

I was less pleased when the authors say that "an appreciable proportion of pigeon fanciers are unwilling to co-operate with studies they fear may ultimately force them to give up their hobby." Working in association with us, Professor J Pepys, of the Cardiothoracic Institute, London, was able to take a random sample from all visitors to our annual Old Comrades Show; such reluctance as there was was in almost every case the not uncommon reaction to being stopped unexpectedly and asked to give a blood sample. The important difference was the confidence that pigeon fanciers had that their results would not be misused.

Unfortunately pigeon fanciers have suffered only too often, particularly from medical officers of health who have made statements to the local press which are extremely prejudicial to pigeon fanciers and do not represent the risks fairly. If you will permit a layman to make a commentary on the difference between pigeon-fanciers' lung and budgerigar-fanciers' lung, I would draw your attention to the selection pressure on racing birds presented by the suppression or loss of weaklings, which would usually include any with chronic symptoms, and the fact that pigeons move from one hygiene-conscious loft to another direct, bypassing the pet shops, of which only the very best could be viewed with satisfaction by either the veterinary or medical profession.

As the chairman of the Racing Pigeon Research Fund for Medical and Veterinary Research I regard it as an important function of my position to advise on pigeon matters beyond the normal knowledge of medica practitioners and I am happy to continue this function. Indeed, the sad news to a fancier that he has got to give up his hobby, which I know is sometimes necessary, often comes better from me than from his general practitioner.

London WC1

Colin A Osman Editor and Managing Director,

Ileostomy or ileorectal anastomosis for ulcerative colitis

Sir,- The article by Mr Peter F Jones and others (3 June, p 1459) was refreshing indeed. The controversy regarding ileorectal anastomosis has in the past been clouded by personal prejudices and overzealous condemnation of the technique by several of the more prominen experts in colorectal surgery. This has resulted in a somewhat pessimistic attitude towards the procedure by many surgeons. Mr Stanley Aylett has been the pioneer of the procedure in the United Kingdom and for those of us who have had the good fortune to see at first hand the excellent functional results in the vast majority of his patients it is good to see the pendulum swinging in a more realistic direction. I continue to be impressed in my own practice with the functional result of ileorectal anastomosis, particularly in young people. I am also encouraged with the increasing evidence that biopsy and histological search for premalignant change in patients with mucosal ulcerative colitis is accurate in being able to predict malignant degeneration and consequently that the ileorectal anastomosis is now safer than before.

I believe your article will help in the correction of the imbalance in surgical opinion regarding ileorectal anastomosis which is long overdue.

D G JAGELMAN

Department of Colon and Rectal Surgery, Cleveland Clinic Foundation,
Cleveland, Ohio

\section{Six months in South Africa}

SIR,-It was a pleasure to read Dr Anne Savage's account of South Africa (29 July, $p$ 329). She is still very much remembered in the Transkei, from where I recently returned after a stay of four and a half months as a "peripatetic" physician. Two hospitals that I visited (Bambizana and Bizana) were entirely without doctors and while staying in one of them for a week I had to perform operations such as caesarean sections, which are not entirely within the armament of a physician. I had learnt to do this, among other new skills, in Holy Cross Hospital, where I was one of seven doctors. This hospital now has joined the ranks of fully commissioned ex-missionary hospitals functioning without one single doctor.

By writing this letter may I please be the "medium for the message": please help the sick of a charming but, from the medical point of view, pathetically abandoned land by offering to work there during one or two annual leaves-as I did. The reward ? A letter such as I have just received from a 22 -year-old hospital clerk in Holy Cross who was comatose for several days with typhoid fever. It reads "We have now absolutely no doctors, I thank God that I have so rapidly recovered. I also thank you for saving my life."

STEPHEN SZANT

Langthorne and Plaistow Hospitals 